\title{
Resolving the 3D Nano-architecture of the Actin Cytoskeleton
}

James Galbraith $^{1}$, Jesse Aaron ${ }^{2}$, Ulrike Boehm ${ }^{2}$, Teng-Leong Chew ${ }^{2}$ and Catherine Galbraith ${ }^{1}$

${ }^{1}$ OHSU, Lake Oswego, Oregon, United States, ${ }^{2}$ HHMI Janelia Research Campus, Ashburn, Virginia, United States

The actin cytoskeleton is a dynamic assembly of polymer chains cross-linked into space-spanning networks and filament bundles. Together, they are responsible for organelle transport, endocytosis, cytokinesis, cell shape, and cell migration. The biochemistry underlying the dynamic assembly - how monomer is added and removed and how polymers are linked and bundled - have largely been identified. However, with the exception of the organization of large fibers and some local networks, the nano-architecture of the cytoskeleton and how it fills the space within the cell remains unclear. As the most abundant intracellular protein, actin itself obscures seeing its organization within the cell. Here we have overcome this visibility obstacle by isolating the location of 100's of millions of individual actin molecules in a single cell. Using a combination of high-density molecular labeling, bright dyes, controlled on/off switching of fluorophore visibility, and 3D interferometric single molecule super-resolution microscopy (iPALM) (Shtengel et al., 2009), we determined the position of each molecule with $16 \mathrm{~nm}$ lateral $(\mathrm{x}, \mathrm{y})$ and $8 \mathrm{~nm}$ axial $(\mathrm{z})$ resolution. We now for the first time visualize individual fibers and resolve the spatial relationship of bundled filaments embedded within dense networks. Our structures answer several long-standing controversies regarding how actin architecture enables cell function, including how the architecture at the leading edge simultaneously facilitates the cellular function of probing for matrix and forming anchors to pull the cell forward. We reveal that the actin cytoskeleton at the front of the cell is a combination of overlapping branched lamellipodial networks and parallel lamella filament bundles that are confined to different axial planes. In axial planes closest to the substrate, there is only a lamellipodial network at the leading edge. In higher axial planes, the lamellipodial network and lamella bundles are intermixed, and the lamella bundles span from the leading edge back through the lamella. At the highest axial planes, there is only a thin layer of lamellipodial actin at the leading edge. Thus, at the leading edge, the lamella fibers are captured within the thicker lamellipodial actin network and the lamella actin fibers only span from the front to the cell interior at an intermediate plane. Treatment with the Arp2/3 inhibitor CK666 decreases the lateral width of the lamellipodium and eliminates the axial capture of the lamella actin between layers of the lamellipodial actin. These single molecule reconstructions of the smallest, densest cytoskeletal network provide the structural framework for understanding how the actin cytoskeleton gives rise to cellular functions.

\section{References}

Shtengel, G. et al. Interferometric fluorescent super-resolution microscopy resolves 3D cellular ultrastructure. Proceedings of the National Academy of Sciences of the United States of America 106, 3125-3130, doi:10.1073/pnas.0813131106 (2009). 